\title{
Corresponding States Correlation of Saturated and Metastable Properties
}

\author{
WEI-GUO DONG' and JOHN H. LIENHARD
}

\section{Heat Transfer and Phase-Change Laboratory, Mechanical Engineering Department, University of Houston, Houston, Texas 77004}

\begin{abstract}
A corresponding states correlation is developed for the $p-v-T$ properties of saturated liquids. It is similar in form to a previous correlation; however it is more accurate in the low pressure, low Pitzer factor range, owing to the incorporation of new information about low Pitzer factor behavior. The problem of creating corresponding states correlations of the spinodal lines and the metastable states is also discussed in the light of new equation of state information. A partial preliminary correlation works particularly well for non-polar molecules in the range of fairly small positive Pitzer factors.
\end{abstract}

On a établi une corrélation du type états correspondants pour les propriétés $p-v-T$ des liquides saturés; elle est de forme semblable à celle d'une corrélation antérieure, mais elle est plus précise à basses pressions et pour de faibles valeurs du facteur de Pitzer, à cause de l'incorporation de renseignements nouveaux sur le comportement pour de faibles valeurs du facteurs de Pitzer. On discute aussi le problème de l'établissement de corrélations de type états correspondants pour les lignes spinodales et pour les états métastables, à la lumière de nouvelles informations sur les équations d'état. Une corrélation partielle préliminaire fonctionne particulièrement bien, dans le cas de molécules non polaires, dans le domaine des facteurs de Pitzer positifs et assez petits.

$\mathrm{T}$ he first corresponding states correlations of the saturation pressures were presented by Pitzer et al. (1955) and by Lydersen et al. (1955). They wrote the Law of Corresponding States ${ }^{2}$ in the form:

$$
T_{r, \text { sat }}=f\left(p_{r}, \text { molecular parameter }\right)
$$

For the molecular parameter, Pitzer used the Pitzer factor, $\omega$,

$$
\omega \equiv-1-\log _{10}\left[p_{r, \text { sal }}\left(T_{r}=0.7\right)\right] \ldots \ldots \ldots \ldots .
$$

and Lydersen et al. used the critical compressibility, $Z_{c}$.

Several investigators subsequently made improved correlations of the vapor pressure curve using $\omega$, which gives superior results. The most accurate was Lee's and Kesler's (1975):

$$
\begin{aligned}
& \ln \left(p_{r, \mathrm{sat}}\right)=5.92714-6.09648 / T_{r}-1.28862 \ln \left(T_{r}\right) \\
& \quad+0.169347 T_{r}^{6}+\omega\left[15.2518-15.6875 / T_{r}\right. \\
& \left.\quad-13.4721 \ln \left(T_{r}\right)+0.43577 T_{r}^{6}\right] \ldots \ldots \ldots \ldots
\end{aligned}
$$

In 1976, Lienhard showed that the Lydersen et al. correlation naturally extrapolated to include the van der Waals saturation curve as $Z_{c}$ approached the van der Waals $Z_{c}$ of $3 / 8$. This was part of a demonstration that the van der Waals fluid was a member of the family of real fluids at the appropriate value of $Z_{c}$. Peck redid this demonstration in 1982 using $\omega$ in place of $Z_{c}$ and obtained even stronger corroboration of Lienhard's result. He found that, as $\omega$ approached the van der Waals value of -0.302 , all reduced thermodynamic properties approached van der Waals values, except where the Law of Corresponding States is known to fail as a consequence of low temperature quantum effects (see, e.g. Watkinson and Lielmezs (1967).)

More recently, Shamsundar and Lienhard (1983) noted that mercury, with an $\omega$ close to -0.302 , is very well represented by van der Waals' equation. The verifiable connection of van der Waals' equation to real fluids provides

'On leave from the Thermal Power Engineering Research Institute, Xi-an, People's Republic of China.

${ }^{2}$ Conventional notation is listed in the Nomenclarure section and not explained in context. important leverage in corresponding states correlations, that we use subsequently.

Nobody has, to our knowledge, attempted to create a corresponding states correlation of properties within the saturation (or coexistence) dome. However, Biney et al. (1985) recently improved on earlier work by Eberhart (1976) and Karimi and Lienhard (1980) in making accurate predictions of liquid spinodal lines. (The spinodal line, or locus of points at which $(\partial p / \partial v)_{T}=0$ in the liquid phase, is illustrated in Figure 1.) Based on the earlier work of Karimi and Lienhard (1980), Biney et al. represented spinodal points as limiting homogeneous nucleation points,

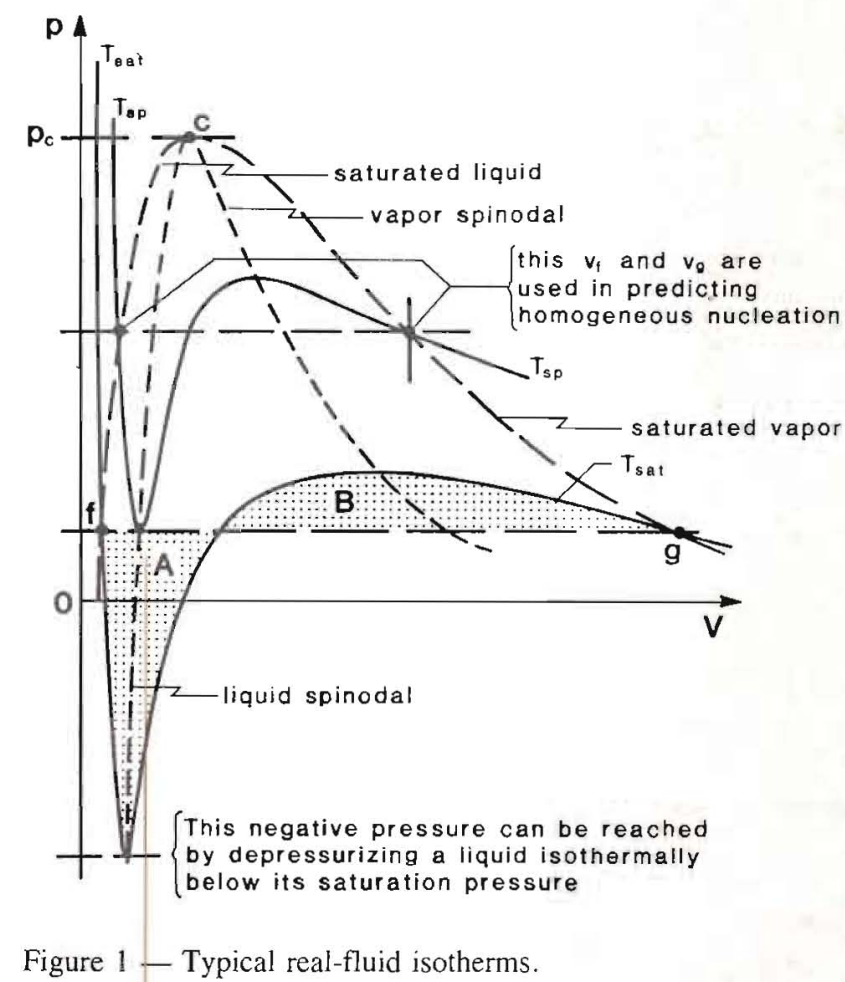




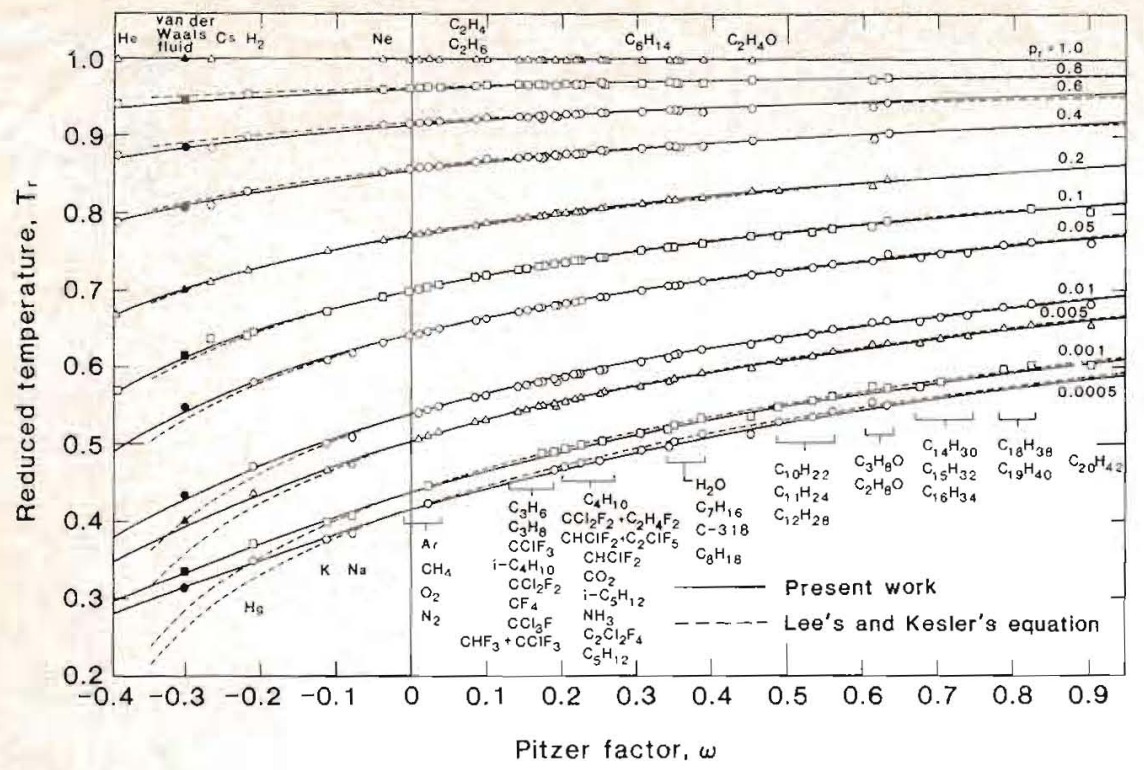

Figure 2 - Correlation of vapor-pressure data for real fluids and the van der Waals fluid.

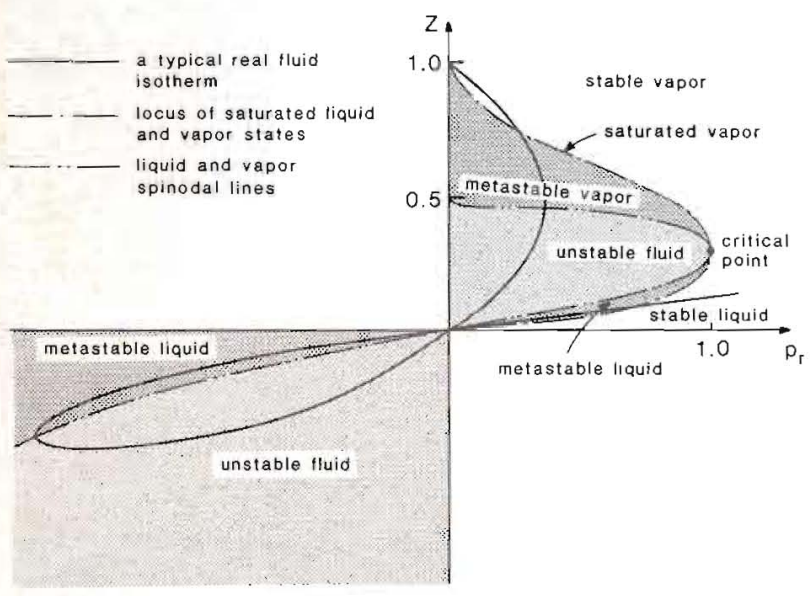

Figure 3-Schematic representation of the metastable and unstable regimes on $Z$-coordinates.

given by:

$$
-\ln j=\frac{16 \pi \sigma^{3}}{3\left(k T_{c}\right)\left[p_{\mathrm{sal}}\left(T_{s p}\right)-p\right]^{2}\left(1-v_{f} / v_{g}\right)^{2}} \ldots \ldots .
$$

where the group $k T_{c}$ replaces the conventional group, $k T$, as Karimi and Lienhard recommend. They showed that the nucleation probability, $j$, normally ranges between $10^{-4}$ and $10^{-5}$.

Biney et al. also showed that when the very general Shamsundar-Murali $p-v-T$ equation of state (see Murali, 1984 or Lienhard et al., 1985)

$$
\frac{p}{p_{\mathrm{sat}}}=1-\frac{\left(v-v_{f}\right)\left(v-v_{m}\right)\left(v-v_{g}\right)}{(v+b)(v+c)(v+d)}
$$

was fitted to real fluids, the results represented the metastable and unstable states - as well as the stable ones with high accuracy. They verified this by substituting the results in van der Waals' (1894) surface tension prediction and accurately obtaining the temperature dependence of the surface tension.

Our present objective is to take a new look at the correlation of stable, metastable, and unstable data on and within the saturation dome. We wish to reconstruct Lee's and Kesler's correlation to include the van der Waals saturation curve. Furthermore, armed with better information

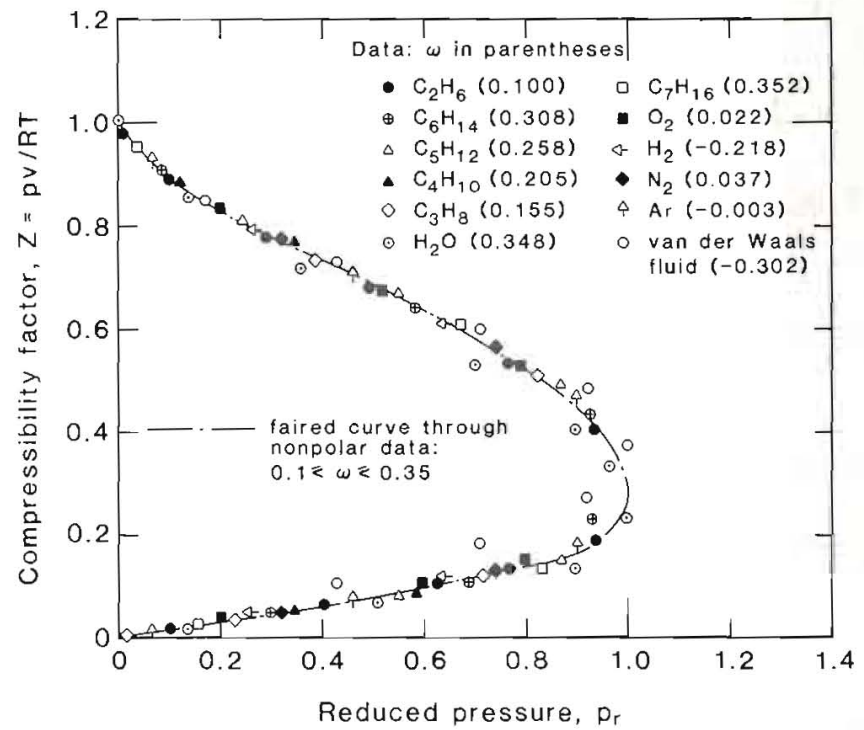

Figure $4-Z$-chart correlation of the vapor-pressure data.

about the spinodal and metastable states, we seek to include them in the correlation.

\section{Correlations}

\section{A. Correlation of Saturated $p-v-T$ data}

The present vapor-pressure correlation is shown in Figure 2. The sources of $(p, T)_{\mathrm{sal}}, \omega, T_{c}$, and $p_{c}$ data used to develop this curve were: Reid et al. (1977), Reynolds (1979), Vargaftik (1975), and the IFC formulation of the properties of water (1968). "Data" for the van der Waals equation calculated by Shamsundar and Lienhard (1983) are also included in the figure. (For clarity, Figure 2 only displays roughly every other isobar used in the correlation.)

An equation similar to Lee's and Kesler's was fitted to 634 data points; however we found it possible to use two fewer terms than they did. The result, which fits all the data with an rms accuracy in $T_{r}$ of \pm 0.42 percent, is

$$
\begin{aligned}
& \ln p_{r . \text { sat }}=5.37270\left(1-1 / T_{r}\right) \\
& \quad+\omega\left(7.49408-11.18177 T_{r}^{3}+3.68769 T_{r}^{6}\right. \\
& \left.\quad+17.92998 \ln T_{r}\right) \ldots \ldots \ldots \ldots \ldots \ldots \ldots
\end{aligned}
$$




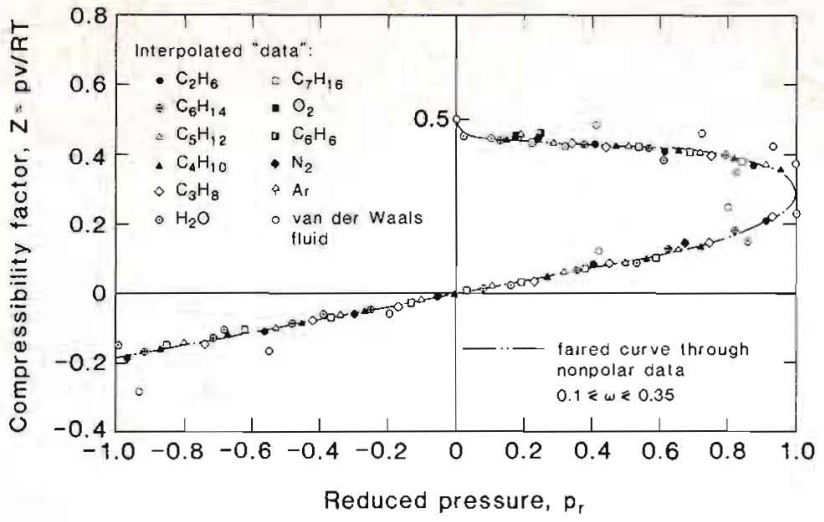

Figure $5-Z$-chart correlation of the vapor and liquid spinodal lines for various substances.

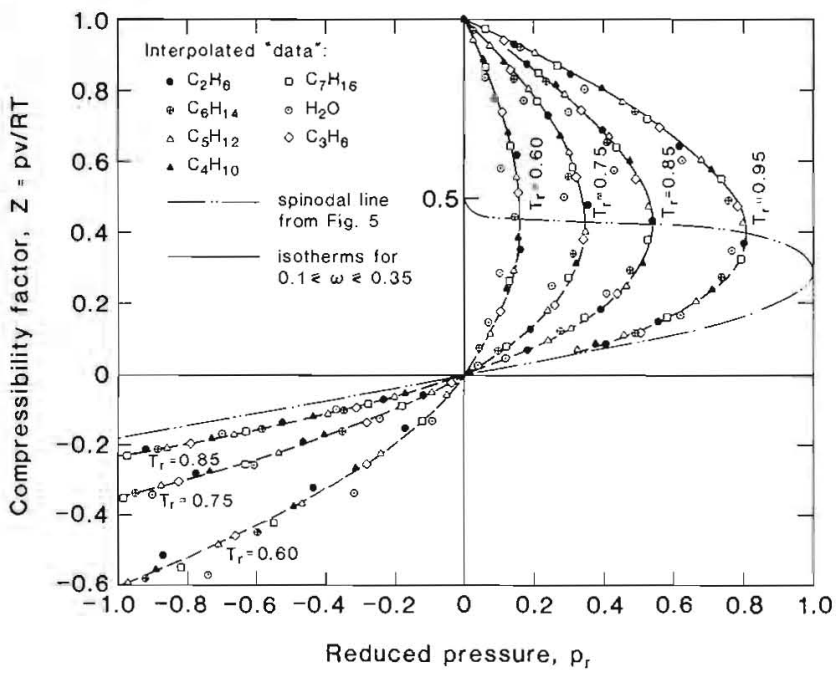

Figure $6-Z$-chart correlation of metastable/unstable isotherms for various substances.

The important feature of Equation (6) is that it is based on data in a larger range of $\omega$ than Equation (3) was, including those substances for which $\omega$ extends well below zero. These include helium $(\omega=-0.391)$, cesium $(\omega=$ $-0.267)$, hydrogen $(\omega=-0.218)$, mercury $(\omega=-0.21)$, potassium ( $\omega=-0.11)$, and sodium $(\omega=-0.078)$, in addition to the van der Waals fluid (which was given double weight in the curve fit.) We have excluded data for helium and hydrogen below $T_{r}=0.6$ where we expect to incur the failures of the Law of Corresponding States that are inevitable when the behavior of these fluids must be dictated by quantum statistics.

Equation (6) gives $p_{r, \text { sar }}\left(T_{r}=1\right)=1$ and it gives Equation (2) at $T_{r}=0.7$. It has not been forced to give a temperatureindependent Riedel factor at the critical point.

The choice between Equations (3) and (6) is a toss-up at high $\omega$ and $T$, except in that Equation (6) is simpler. However, Equation (6) is far superior at low values of $\omega$ and $T$.

\section{B. Corresponding states Correlations of metastable PROPERTIES}

Figure 3 is a schematic diagram that shows how the $p-v-T$ surface (Figure 1) maps into generalized compressibility coordinates: $Z=Z\left(p_{r}, T_{r}\right)$. The various regimes are indicated on it for the readers convenience.

Figures 4 and 5 show the vapor pressure and spinodal line

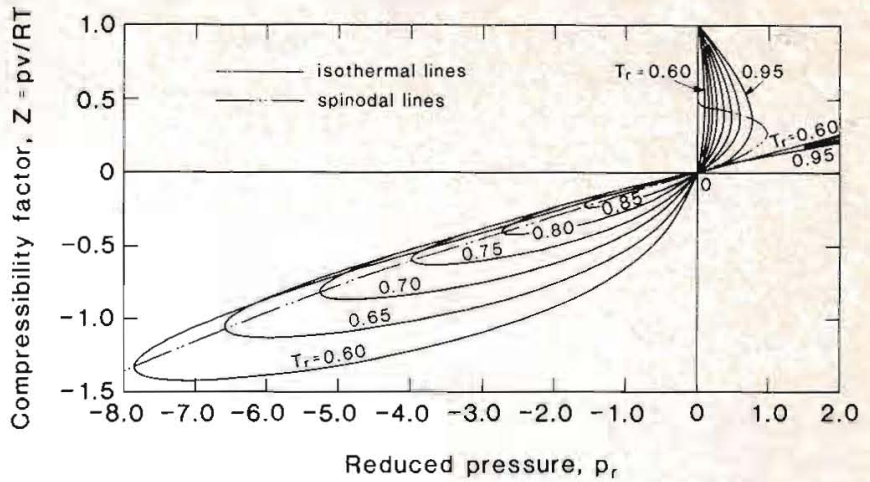

Figure 7 - Form of the Z-chart for a large range of metastable/unstable properties - based on water.

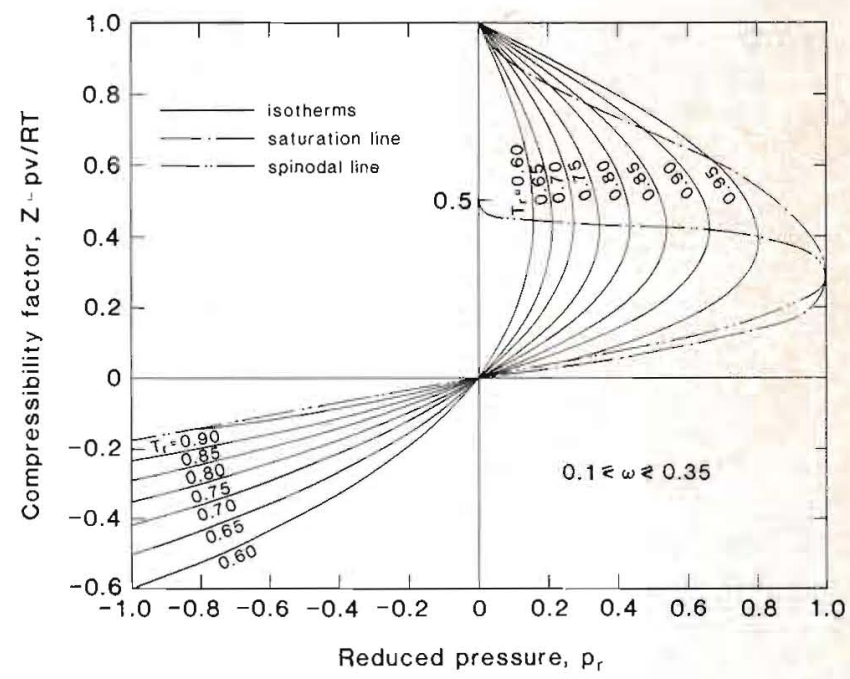

Figure 8 - The $Z$-chart for metastable, unstable, and saturated fluids. Accurate for non-polar fluids in the range $0.10 \overline{<} \omega \overline{<} 0.35$.

results (including van der Waals values) on $Z$ coordinates, and Figure 6 shows several metastable isotherms. The vapor pressure data are those upon which Equation (6) was based. The spinodal lines (which are consistent with Equation (4)) and the isotherms were generated by the curve fits of the Shamsundar-Murali equation of state executed by Biney et al. All three figures verify that the spread of data in $\omega$ is generally not great on these coordinates - particularly for non-polar molecules with relatively low positive $\omega$ 's. The scatter is predictably larger on the liquid side than on the gaseous side, and it increases strongly toward the critical point.

The reader should note that we extrapolate the vapor spinodal line to $Z=0.5$ at low pressure. This result, noted by Karimi and Lienhard (1979), stems from the fact that one may write any equation of state in virial form:

$$
p=R T / v+B / v^{2}+C / v^{3}+
$$

One may then differentiate it and set the result equal to zero on the spinodal line. At low pressures in the vapor region we may further drop terms of degree beyond $v^{-2}$. We solve the result:

$$
(\partial p / \partial v)_{T}=-R T / v^{2}-2 B / v^{3}=0
$$

for $B=-R T V / 2$ which, substituted into the low pressure virial, gives:

$$
p=R T / v-R T v / 2 v^{2}=R T / 2 v \text { or } Z=1 / 2 .
$$


Figures 4,5 , and 6 thus suggest that it is feasible to create a $Z$-chart in the metastable and unstable regimes, particularly for a restrictive range of $\omega$ 's - just as is done in the stable ranges. Figures 7 and 8 are such Z-charts. However, Figure 7 is only based on the Biney et al. curve fits for water (with $\omega=0.348$ ) which - while not the most general case - is the best documented and which best satisfied all of their tests for validity. Water is slightly displaced from the center of the data scatter in Figures 4, 5, and 6. Thus Figure 7 merely displays the qualitative features of a larger $p_{r}$ range - beyond the range where Biney et al. claimed good accuracy.

The curves in Figure 8, on the other hand, are limited to the ranges in which pentane (with $\omega=0.258$ ) represents the remaining data well. They are "best-fit" curves through the data for the simple-substances and, while they do not strictly represent pentane, they never stray far from it. We recommend Figure 8 as the corresponding states correlation for the region within the saturation dome. It represents at least all of the nonpolar substances that we present within \pm 0.02 in $Z$, in the ranges:

$$
0.10<\omega<0.35, \quad p_{r \text {. liquid }}<0.8, \quad p_{r, \text { vapor }}<0.9
$$

Figure 8 can still be used outside these ranges, but the accuracy can be expected to fall off further away from them.

Figure 8 does not include any metastable liquid data. While these data can also be correlated well within \pm 0.02 in $Z$ over large ranges: (a) the scatter becomes too large in comparison with the small absolute value of $Z$; (b) there is very little separation among lines of constant temperature in this range; and (c) one would have to include the Pitzer factor in such a correlation.

\section{Conclusions}

1. Equation (6) is recommended for the prediction of the vapor-pressure curve of any substance.

2. The general form of the Z-chart for states within the saturation dome is given in Figure 7.

3. Figure 8 is the subset of Figure 7 that is the recommended $Z$-chart. It is most accurate for use with non-polar substances, with low positive Pitzer factors, not too close to the critical point.

\section{Nomenclature}

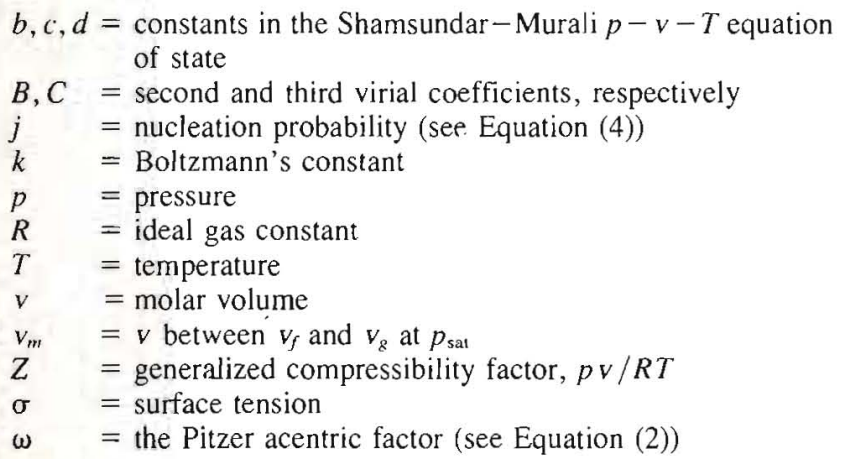

\section{General superscripts and subscripts}

$c \quad=$ a property at the critical point

$f, g=$ saturated liquid or vapor properties $r \quad=$ a "reduced" property (e.g. $\left.X_{r} \equiv X / X_{c}\right)$

sat $=$ a property at a saturation condition

$\mathrm{sp} \quad=$ a property at a spinodal point

\section{References}

"The 1968 IFC Formulation for Scientific and General Use. A Formulation of the Thermodynamic Properties of Ordinary Water Substance". Prepared by the International Formulation Committee (IFC), Available from the Secretariat of the International Conference on the Properties of Steam, ASME, New York, (1968).

Biney, P. O., W-g. Dong and J. H. Lienhard, "Use of a Cubic Equation to Predict Surface Tension and Spinodal Limits", AIChE/ASME 23rd Nat'l. Heat Transfer Conf., Denver (Aug. 4-7, 1985).

Eberhart, J. G., "The Thermodynamic and the Kinetic Limits of Superheat of a Liquid", J. Colloid. and Interface Sci., 56 (2), 262-269 (1976).

Karimi, A. and J. H. Lienhard, "Toward a Fundamental Equation for Water in the Metastable States", High Temperatures - High Pressures, 11, 511-517 (1979).

Karimi, A. and J. H. Lienhard, "Homogeneous Nucleation and the Spinodal Line", J. Heat Transfer, 102(2), 457-460 (1980)

Lee, B. I. and M. G. Kesler, "A Generalized Thermodynamic Correlation Based on Three-Parameter Corresponding States", AIChE J., 21(3), 510-527 (1975).

Lienhard, J. H., "Relation Between van der Waals Fluid and Real Substances", Iranian J. Sci. and Tech., 5, 111-116 (1976).

Lienhard, J. H., N. Shamsundar and P. O. Biney, "Spinodal Lines and Equations of State", A Review, Nuclear Sci. and Engr., (in press).

Lydersen, A. A., R. A. Greenkorn and O. A. Hougen, "Generalized Thermodynamic Properties of Pure Fluids", Univ. of Wisc., College of Engr. Rept. 4, (October 1955).

Murali, C. S., Cubic Equation of State for Water, Hydrocarbons, and Liquid Metals, MSME thesis, Dept. of Mech. Engr., Univ. of Houston, 1984.

Peck, R. E., "The Assimilation of van der Waals Equation in the Corresponding States Family", Can. J. Chem. Eng., 60, 446-449 (1982)

Pitzer, K. S., D. Z. Lippman, R. F. Curl, C. M. Huggins and D. E. Peterson, "The Volumetric and Thermodynamic Properties of Fluids. II. Compressibility Factor, Vapor Pressure and Entropy of Vaporization", J. Am. Chem. Soc., 77, 3433-3440 (1955).

Reynolds, W. C., Thermodynamic Properties in SI, Department of Mechanical Engineering, Stanford University, (1979).

Reid, R. C., J. M. Prausnitz and T. K. Sherwood, The Properties of Gases and Liquids, McGraw-Hil] Book Co., New York, Appendix A (1977)

Shamsundar, N. and J. H. Lienhard, "Saturation and Metastable Properties of the van der Waals Fluid", Can. J. Chem. Eng., 61, $876-880$ (1983).

van der Waals, J. D., "Thermodynamische Theorie der Kapillarität unter Voraussetzung stetiger Dichteänderung", Zeit. Phys. Chemie, 13, 657-725 (1894). [Also published as "The Thermodynamic Theory of Capillarity Under the Hypothesis of a Continuous Variation of Density", (tr. by J. S. Rowlinson) J. Chem. Phys., 20(2), 197-244, (1979)].

Vargaftik, N. B., Tables on the Thermophysical Properties of Liquids and Gases, 2nd ed., Hemisphere Publishing Corp. Washington, D.C., (1975).

Watkinson, A. P. and J. Lielmezs, "Corresponding States principle and Surface Tension”, J. Chem. Phys., 47, 1158 (1967).

Manuscript received April 18, 1985; revised manuscript received July 5, 1985; accepted for publication July 8, 1985. 